\title{
Die Blasiertheit der Städter*innen - Vom routinierten Umgang mit alltäglicher Komplexität
}

\author{
Nina Berding \\ Research Centre of the University of Siegen "Shaping the future", Siegen, Germany \\ Correspondence: Nina Berding (nina.berding@posteo.de)
}

Received: 31 July 2018 - Revised: 6 May 2019 - Accepted: 8 May 2019 - Published: 6 June 2019

\begin{abstract}
Kurzfassung. In everyday life, people will act pragmatically. Continuing their routines as they move through the urban space. To enable people to deal with the everyday complexities and diversity of everyday life, people develop routines to help simplify their existence. These daily routines contain distinctive processes and a certain "blasé attitude" to normal or trivial behavior. Using the example of my ethnographic research at DüsseldorfOberbilk it could be argued that this kind of behavior repertoire is crucial for successfully dealing with diversity and complexity of urban life.
\end{abstract}

\section{Einleitung}

„[... [ Es kommt nun quer die Dreieckstraße. Eine Art Dreieck, in der Mitte befindet sich ein trostloser Platz und drum herum auch relativ trostlose Häuserfassaden. In den Erdgeschossen sehen wir einen Metzger, eine Shisha Bar, einen Waschsalon und einen Friseur. Es sind kaum Menschen anzutreffen; nur vereinzelt stehen Männer einfach so herum. Frauen sieht man kaum. Das mag auch daran liegen, dass die Dreiecksstraße direkt zum Bahndamm führt, der vom Platz aus ca. 100 Meter entfernt liegt. Bei einem Blick in die Seitenstraße, die zum Bahndamm führt, sehen wir Sexshops und kleine Ladenlokale mit verklebten Fensterscheiben. Wir gehen wieder zurück zum ,Dreiecksplatz ‘ und folgen der Lessingstraße bis zum Lessingplatz. Um den Lessingplatz herum stehen überwiegend bunte und hohe, gepflegt aussehende Altbauten. Gekrönt wird der Platz von der Apollinariskirche. Der Lessingplatz selber ist ein riesiger mit Bäumen umzäunter Platz, der viele Verweilmöglichkeiten bietet. Im Moment ist er aber still; auf dem Spielplatz spielen wenige Kinder und wir sehen wenige Erwachsene auf den Bänken sitzen. Der Bolzplatz ist leer. Nur am Nachbarschaftshaus, das bunt gestaltet wurde, sitzt eine große Männergruppe mit
Bierflaschen auf den Bänken. Davor ist direkt ein Marktstand aufgebaut... "(FN, 17. April 2015). ${ }^{1}$

Düsseldorf-Oberbilk ist ein bunter, durch unterschiedliche Lebensstile und -weisen geprägter ehemaliger Industriestadtteil: Rotlicht am Bahndamm und moderne Verwaltung am Bertha-von-Suttner-Platz, Cafés und Restaurants neben Supermarktketten auf der Kölner Straße, angesagte ShishaBars entlang der Ellerstraße und das traditionsreiche UerigeBrauhaus am Oberbilker Markt, der Volksgarten als grüne Lunge des Quartiers und der nie abschwellende Verkehrsstrom auf der Kruppstraße, heruntergekommene 50er-JahreZweckbauten und prachtvoll renovierte Altbauten am Lessingplatz. Oberbilk ist vieles und doch für jeden/jede etwas anderes, ein Ort, an dem unterschiedliche Menschen mitund nebeneinander leben, ein Ort, der aufgrund der unterschiedlichen Lebenslagen seiner Bewohner*innen aber auch umkämpft ist. Schon immer war Oberbilk durch Diversität und Internationalität geprägt. Die hier ansässigen großen Industrieunternehmen zogen ab den 1850er Jahren Arbeiter*innen von weit her an, die im unmittelbaren Umfeld der Unternehmen günstigen Wohnraum fanden. Als attrak-

\footnotetext{
${ }^{1}$ Im Rahmen meiner Forschung im Stadtteil DüsseldorfOberbilk habe ich neben klassischen Feldbeobachtungsprotokollen (FB) auch Feldnotizen (FN) verfasst: Szenische, kurze Beobachtungen, Gedankengänge, kurze Interaktionen oder Begegnungen, die im Rahmen des Forschungsprozesses entstanden sind und die ich nicht zu längeren Feldprotokollen verdichtet habe.
} 
tiver Industriestandort wuchs der Stadtteil in einem rasanten Tempo zu einem gewachsenen Arbeiterquartier heran. Lebten 1850 nur knapp 1000 Einwohner*innen in Oberbilk, so waren es 1871 bereits 5204 Einwohner*innen. Auch in der Folge wuchs Oberbilk schneller als die Stadt Düsseldorf insgesamt: Von 1871 bis 1885 stieg die Einwohnerzahl in der Gesamtstadt um $66 \%$, in Oberbilk hingegen um $127 \%$ auf ca. 11800 Einwohner*innen (vgl. Glebe, 1998:64). Ohne Zuwanderung - erst aus den Eifeldörfern, dann aus den französisch-wallonischen und polnisch-böhmischen Gebieten und ab den 1950er-Jahren aus dem europäischen und globalen Süden - wäre Oberbilk nicht der Stadtteil geworden, der er heute ist (vgl. Glebe, 1998:64).

Die Industrie- und Mobilitätsgeschichte prägt bis heute die Bau- und Siedlungsstruktur Oberbilks sowie die Zusammensetzung der Bevölkerung: Diversität ist konstitutiv für den Stadtteil wie auch für die Stadt Düsseldorf insgesamt. Überhaupt gilt, dass Diversität die Grundkonstituente städtischen Lebens ist. Und doch wird gerade der Normalfall der Diversität häufig als Gefahr für das Zusammenleben in den Städten problematisiert, so auch im Falle Oberbilks. Die Geschichte Oberbilks als ehemals proletarisches Arbeiterquartier und die damit verbundene Heterogenität haben dem Quartier immer wieder ein randständiges Image verpasst, das vor allem das Nicht-Funktionieren des Zusammenlebens fokussiert und bestimmte Newcomer*innengruppen ${ }^{2}$ als Problemauslöser verbesondert (vgl. Berding, 2018a). Als ,,randständiger“ Arbeiterstadtteil hinter dem Bahnhof, als ,Parallelgesellschaft" und Wohnort sogenannter ,nordafrikanischer Straftäter" wurde das Zusammenleben in Oberbilk in der medialen Öffentlichkeit immer wieder diskutiert und infrage gestellt (vgl. u.a. Bürger- und Schützenzeitung, 19271939; RP, 2016; FAZ, 2016; Express, 2016 u.v.m.). Diese Sichtweise auf das Zusammenleben in ehemals proletarischen Stadtteilen hängt nicht zuletzt auch mit einer langjährigen angenommenen Verfallssemantik der Städte zusammen: ${ }^{3}$ „,Die Heterogenität der Städte wird in Deutschland per se als Niedergang betrachtet - der Blick richtet sich kaum einmal auf das, was funktioniert, sondern grundsätzlich auf das Problematische“ (Terkessidis, 2005).

Im folgenden Beitrag möchte ich Ergebnisse aus meiner ethnografischen Forschung im Stadtteil Düsseldorf-Oberbilk

\footnotetext{
${ }^{2}$ In der vorliegenden Arbeit verwende ich den Begriff Newcomer*innen für Menschen die kürzlich landes-, stadt- oder quartiersweite Umzüge vollzogen haben. Um Menschen nicht auf ihr „migrantisch sein“ festzuschreiben oder ihnen ,Wurzeln“ anzudichten, distanziere ich mich in der vorliegenden Arbeit von Begriffen wie beispielsweise „Person mit Migrationshintergrund“.

${ }^{3}$ Obwohl die Großstadt immer wieder auch positiv beschrieben wurde, prägte dennoch von Beginn an eine Verfallssemantik (Bukow and Yildiz, 2002:11) die Diskussion um die Stadt, die bis heute nachwirkt. Die aktuellen Debatten um die „Spaltung der Städte“ oder die Angst vor „Parallelgesellschaften“ sowie die Etikettierung bestimmter Orte als ,soziale Brennpunkte“ machen die konfliktorientierte Sichtweise auf das Zusammenleben in der Stadt deutlich.
}

vorstellen. ${ }^{4}$ Im Rahmen meiner sechzehn monatigen Feldforschung (2015-2016) habe ich anhand von (teilnehmenden) Beobachtungen, Wahrnehmungsspaziergängen, leitfadengestützten, offen-narrativen Interviews $(N=28)$ sowie alltäglichen Gesprächen im öffentlichen Raum, die ich mit verschiedenen lokalen Akteur*innen (Quartiersbewohner*innen, Sozialarbeiter*innen, Vereins- und Institutionsleiter*innen, Pädagog*innen, Integrationsexpert*innen) geführt habe, herausgearbeitet, wie Menschen in einem dichten und gemischten Quartier alltagpraktisch zusammenleben. Das gesamte Forschungsmaterial wurde im Rahmen der grounded theory ausgewertet.

Mein Ziel war es, zu untersuchen, wie es die Quartiersbewohner*innen schaffen, sich trotz unterschiedlicher Interessen in der Nutzung und Gestaltung der öffentlichen Räume miteinander zu arrangieren, bzw. welche Strategien sie anwenden, um in ihrem Alltag relativ störungsfrei miteinander auskommen zu können. Aus einer - sofern mir möglich neutralen Perspektive heraus, war es mein Ziel die „Niederungen des Alltags“ (Yildiz, 2014) zu fokussieren und besonders herauszuarbeiten, was im Zusammenleben und im alltäglichen Miteinander des Menschen im Quartier funktioniert. Diesen Fragestellungen liegt die These zugrunde, dass Menschen zunächst einmal daran interessiert sind, ihren Alltag störungsfrei zu durchlaufen. Ich bin in meiner Forschung also zunächst von der Grundannahme ausgegangen, dass Menschen am Funktionieren des Alltags und nicht an der Zerstörung des Alltags interessiert sind. Betrachtet man die Potenziale, die in den alltäglichen Routinen der Stadtbewohner*innen und in der damit einhergehenden blasierten Haltung gegenüber alltäglichen Irritationen stecken, so zeigt sich, dass die Bewohner*innen in der Lage sind, alltägliche Probleme im Miteinander zu lösen - sofern man sie lässt. Im Rahmen der Forschung hat sich gezeigt, dass die Quartiersbewohner*innen Verfahren anwenden, um mit dem komplexen und ambivalenten, von Irritationen und Störungen durchsetzten Alltag umzugehen. Diese Verfahren habe ich als Vielfaltsverarbeitungsroutinen identifiziert. Sie ähneln dem, was Simmel (2006 [1903]) vor mehr als hundert Jahren bereits als städtische Blasiertheit beschrieben hat und was seitdem aus unterschiedlichen Richtungen als ,resignierende Humanität“ (Bahrdt, 1956), „höfliche Nichtbeachtung “ (Goffmann, 1971) oder „urbane Kompetenz“ (Lindner, 2002) und urbane „Distanziertheit“ (Nassehi, 2017a) oder mit der Figur des „neutralen Fremden“ (Radke, 1991), beschrieben wurde.

Die Quartiersbewohner*innen versuchen, sich mit Hilfe ihrer individuellen Routinen im komplexen Alltag zu bewegen, Störungen und Irritationen zu bewältigen und sich selbst gesellschaftlich zu platzieren. Es lässt sich deshalb

\footnotetext{
${ }^{4}$ Die gesamten Ergebnisse der ethnografischen Studie sind in der noch unveröffentlichten Dissertation: „Der urbane Raum Lessingplatz in Düsseldorf-Oberbilk. Städtischen Alltag arrangieren: Eine ethnografische Studie über ganz alltägliche Konflikte im Umgang mit urbaner Vielfalt"“(vgl. Berding, 2018b) zu finden.
} 
sagen, dass alle Quartiersbewohner*innen gleich im anders sein sind - egal, ob sie arm, reich, Newcomer*in oder alteingesessen sind. Sie sind gleich, weil sie gleichermaßen routinierte Verhaltensweisen anwenden, um mit gesellschaftlicher Komplexität umzugehen und anders, weil sich ihre Routinen voneinander unterscheiden. Dies bedeutet zunächst einmal, dass alle Quartiersbewohner*innen zum Zusammenleben im Quartier beitragen, da sie routinierte Wege suchen, um sich im komplexen Alltag miteinander zu arrangieren und die eigenen Wünsche und Bedürfnisse in das Gesamtgeflecht einzufassen. Der Blick auf das pragmatisch-orientierte Handeln der Menschen im Alltag eröffnet eine Sichtweise auf Prozesse des Zusammenlebens fernab vorgefertigter, stigmatisierender und migrantologischer Zuschreibungen, da derartige Etikettierungen für das pragmatisch-orientierte Funktionieren des Alltag konstitutiv belanglos sind.

\section{Forschungsstand: Die diversitätsgrundierte Stadt}

Es sind vor allem zwei Perspektiven, die im wissenschaftlichen und gesellschaftstheoretischen Diskurs immer wieder aufgegriffen werden, wenn es darum geht, Diversität in den Städten und damit verbundene Prozesse des städtischen Zusammenlebens zu bewerten. Betrachtete die stadtsoziologische Forschung das Zusammenleben der Menschen in den Städten, so wurde auf der einen Seite lange Zeit die „Krise der Stadt“ (u.a. Heitmeyer, 1998; Heckmann, 1998; aber auch ältere Studien etwa von Wirth, 1974) ausgerufen. Es wurden vermeintlich desintegrative Aspekte, wie Armut, „Ethnie“ und abweichendes Verhalten, womit häufig die Distanzierung oder Distinktion bestimmter ,ethnischer“ Gruppen gemeint war, herausgearbeitet, die, so die Befürchtung, letztlich zur Herausbildung abgehängter Stadtteile, zu Parallelgesellschaften und Ghettos führen (Heitmeyer, 1998). Mit dem auch heute noch die ,urbanistische Wissensproduktion“ (Tsianos, 2013:58) prägenden Narrativ der ,desintegrativen Ethnizität“" (Tsianos, 2013:32) wurden und werden kulturelle Unterschiede immer wieder für sozialräumliche Problemlagen verantwortlich gemacht, die beseitigt werden müssen (vgl. Mecheril et al., 2010; Ronneberger and Tsianos, 2009; Yildiz, 2014; Bukow et al., 2001). Dass die Zurschaustellung der angeblich desintegrativen Aspekte und die daraus abgeleiteten Sofortmaßnahmen und Förderprogramme häufig selbst desintegrative Folgen für die betroffenen Stadtteile hatten, wurde dabei allerdings oftmals übersehen (vgl. hierzu kritisch Lanz, 2007, 2016; Häußermann, 2006; Ottersbach and Yildiz, 2004; Ronneberger and Tsianos, 2009).

Der desintegrativen Kraft der Städte wird in stadtsoziologischen Arbeiten auf der anderen Seite aber auch immer wieder das integrative Potenzial diversitätsgeprägter Quartiere entgegengestellt und Diversität mitunter als Heilmittel für ein gelungenes Miteinander auf verschiedenen Ebenen zelebriert (vgl. u.a. Vaiou und Kalandide, 2009; Sennett, 2013; Wiesemann, 2015; Feldtkeller, 2012). Diskussionen um die richtige soziale und kulturelle Mischung in Stadtquartieren oder um geeignete öffentliche Orte, an denen sich Menschen unterschiedlicher sozio-kultureller Herkunft im Alltag begegnen und im besten Falle in Kontakt treten können, sind aus dieser Perspektive zentrale in der Debatte um Vielfalt in der Stadt. $\mathrm{Ob}$ derartige Mischungsszenerien allerdings nicht auf einer verzerrten und normativistischen Wahrnehmung des urbanen Zusammenlebens fußen, kann an dieser Stelle nicht vertiefend diskutiert werden. Fakt ist jedoch, dass sich die Diskussion um eine verträgliche soziale Mischung bis heute trägt und im Spannungsfeld zwischen Mythos, Utopie, Ideologie und Wirklichkeit diskutiert wird (vgl. u.a. Schneider, 2016; Dangschat, 2013; Harlander and Kuhn, 2012; IBA-Studie, 2013 u.v.m.).

Weniger - aktuell jedoch zumindest in der wissenschaftlichen Forschung wachsende - Aufmerksamkeit findet eine neutrale Sichtweise auf Vielfalt in der Stadt, in der Konflikte, Ambivalenzen und Irritationen als konstitutiv wahrgenommen werden und städtisches Zusammenleben pragmatisch betrachtet wird (vgl. Yildiz, 2018; Heide et al., 2010; Dika et al., 2011). Diese Perspektive vermeidet die - vor allem aus den Extrempositionen Diversität $=$ gefährlich/Diversität $=$ unproblematisch abgeleitete Holzschnittartigkeit einiger Analysen und der daraus resultierenden Ableitungen und Handlungsempfehlungen.

Die postmigrantische Perspektive setzt meines Erachtens an dieser Neutralität an, in dem sie Vielfalt nicht als ein besonderes Phänomen, sondern als trivialen Bestandteil von Städten und Quartieren hervorhebt. Aus einer postmigrantischen Forschungsperspektive heraus bleibt das Quartier ein zentraler Bezugspunkt, um gesellschaftliches Zusammenleben erforschen zu können (vgl. u. a. Yildiz, 2018; Römhild, 2014, 2011; Römhild und Bergmann, 2003; Baumgärtner, 2009). Im vorliegenden Beitrag wird das Quartier nicht in einem geschlossen-euklidischen Sinne, sondern als unumgrenztes Produkt sozialen Handels verstanden, das gesellschaftliche Strukturen widerspiegelt und soziale Interaktionen wiederum gleichermaßen beeinflusst. Demzufolge existiert das Quartier nicht per se, sondern wird durch individuelle und kollektive Aneignungsprozesse immer wieder neu hervorgebracht und ausgehandelt. Ein Quartier ist also für jeden/jede etwas anderes, hat für jeden/jede unterschiedliche Begrenzungen. ${ }^{5}$ Aus einer postmigrantischen Perspektive auf das gesellschaftliche Zusammenleben im Quartier stehen jedoch nicht mehr die vermeintlich desintegrativen Verhaltensweisen und sozioökonomischen Charakteristika bestimmter gesellschaftlicher (meist ethnisch definierter) Gruppen, sondern das konkret erlebbare Alltagsleben der Menschen im Mittelpunkt des Forschungsinteresses, da sich im Alltagsleben die vielfältigen, miteinander verwobe-

\footnotetext{
${ }^{5}$ Im Zuge meiner Forschung hat sich immer wieder gezeigt, dass Oberbilk ein stark fragmentiertes Quartier ist, sodass die Bewohner*innen in erster Linie von unterschiedlichen Kiezen oder Kleinquartieren innerhalb des Gesamtquartiers ausgehen.
} 
nen und aufeinander bezogenen Handlungen der Quartiersbewohner*innen situativ von Moment-zu-Moment manifestieren.

Eine postmigrantische Betrachtung des alltäglichen $\mathrm{Zu}-$ sammenlebens fokussiert damit weniger darauf, was im $\mathrm{Zu}-$ sammenleben der Menschen im Quartier nicht funktioniert als vielmehr darauf, was alltagspraktisch und routiniert funktioniert (vgl. u.a. Foroutan, 2016; Yildiz and Hill, 2015). Sie nimmt die Potenziale in den Blick, die sich in den situativen gesellschaftlichen Aushandlungsprozessen der Menschen zeigen, statt die Konflikte in den Vordergrund zu rücken. Dies bedeutet nicht, dass Konflikte verharmlost oder verkleinert werden sollen. Konflikte gehören zum Alltag dazu. Sie sind konstitutiv für städtisches bzw. gesellschaftliches Leben überhaupt und müssen auf irgendeine Art und Weise verhandelt werden. Außerdem gibt die postmigrantische Perspektive mit ihrem neutralen und nicht verbesondernden Blick auf Migration und Diversität Aufschluss über bestehende hegemoniale Deutungshoheiten: Die Auswirkungen eines tradierten Diversitäts- und Migrationsregime, also tradierte Formen von Alltagsrassismus und bestehende Sündenbockideologien, auf die zur Exklusion bestimmter Gruppen immer wieder zurückgegriffen wird, kommen aus dieser Perspektive besonders deutlich zum Vorschein (vgl. Oltmer, 2017).

\section{Blasiertheit als inklusiver Verhaltensstil}

Simmel hat in „Die Großstädte und das Geistesleben“ (1903) den Verhaltensstil der Blasiertheit herausgearbeitet, den Stadtmenschen anwenden, um sich im städtischen Alltag trotz allgegenwärtiger Diversität und auf sie einprasselnder Irritationen unbeschwert bewegen zu können. Das einzelne Individuum muss eine blasierte Haltung gegenüber den Reizen des Großstadtlebens entwickeln, da diese Haltung die Verarbeitung des tagtäglich Erlebten erst ermöglicht. Blasiertheit definiert Simmel folgendermaßen: „Das Wesen der Blasiertheit ist die Abstumpfung gegen die Unterschiede der Dinge, nicht in dem Sinne, daß sie nicht wahrgenommen würden, wie von dem Stumpfsinnigen, sondern so, daß die Bedeutung und der Wert der Unterschiede der Dinge und damit der Dinge selbst als nichtig empfunden wird" (Simmel, 2006 [1903]:121). Blasiertes Verhalten dient dem Individuum als Schutzpanzer, um den Alltag entsprechend individuell organisieren zu können und nicht an einer psychischen Überbelastung durch Neues zu zerbrechen. Die diversen Lebensformen, die auf das Individuum im städtischen Alltag selbstverständlich einwirken, kann das Individuum, würde es sich in all die Lebensformen emotional involvieren, die um es herum selbst im kleinräumigen Alltag existieren, gar nicht bewältigen: „Wenn der fortwährenden äußeren Berührung mit unzähligen Menschen so viele innere Reaktionen antworten sollten [...] so würde man sich innerlich völlig atomisieren und in eine ganz unausdenkbare seelische Ver- fassung geraten“ (Simmel, 2006 [1903]:122f.). Blasiertheit im Simmelschen Sinne betrachte ich damit als eine Form der Gleichgültigkeit und Reserviertheit gegenüber fremden Menschen, Dingen und Verhaltensweisen. Gleichgültig sein bedeutet dabei nicht den Problemen und Bedürfnissen der Anderen gegenüber abgestumpft zu sein. Eine blasierte Haltung ist keine Verhaltensweise durch die sich die Eine über den Anderen erhebt, sondern ein neutraler, überlebenswichtiger Verhaltensstil, den Stadtmenschen anwenden, um den facettenreichen, aufgrund der Vielfalt von Unterschiedlichem oft überfordernden, städtischen Alltag bewältigen zu können. Blasiertheit bedeutet also schlichtweg, den Unterschieden zwischen den Individuen sachlich zu begegnen. Damit deutet die von Simmel psychologisierende Betrachtung des großstädtischen Alltagslebens vor allem auf ,zivilisatorische Errungenschaften wie individuelle Freiheit, soziale Vielfalt und gesellschaftliche Toleranz" (Schöller-Schwedes, 2008:653) hin, die im Verhalten des Stadtmenschen sichtbar werden. Es deutet die Fähigkeit der/des Einzelnen an, sich an einen globalisierten und mobilitätsdurchdrungenen Alltag anzupassen.

Simmels pragmatische Sicht auf das Zusammenleben der Menschen in den Städten ist bis heute ein wichtiger Bezugspunkt der Stadtsoziologie, auch wenn Simmels Blasiertheit in der Bewertung von Prozessen städtischen Zusammenlebens wenig Berücksichtigung erhält. Armin Nassehi hat die mit Simmel eingeleitete pragmatische Sichtweise auf das $\mathrm{Zu}$ sammenleben aufgegriffen und die Blasiertheit als eine Form der individuellen Distanz im Alltag als eine positive Ressource hervorgehoben (vgl. etwa Nassehi, 1997, 2017b: „Distanz ist in unserer urbanen Welt eine wichtige Ressource geworden, weil wir den ganzen Tag mit Fremden zu tun haben. Gemeinsam einsam zu sein ist keine Bedrohung, sondern Voraussetzung für das Zusammenleben in der modernen Gesellschaft" (Nassehi, 2017a). Nassehis Distanzbegriff greift meines Erachtens zentrale Aspekte der Simmelschen Blasiertheit auf. Denn auch Nassehi meint mit Distanz eine individuelle pragmatische Grundhaltung im Alltag, sich je nach Situation von Menschen und Artefakten abgrenzen zu können oder sich ihnen eben - je nach individuellem Bedarf - zu nähern.

In gegenwärtigen Gesellschaften muss die blasierte Verhaltensweise eine noch viel stärkere Ausprägung aufweisen, da die Diversität in urban-städtischen Agglomerationen immer stärker zunimmt, sich also immer mehr „Fremde“ im Alltag flüchtig begegnen. Sei es das aneinander Vorbeigehen auf der Straße, das Anstehen beim Bäcker oder auch das Ignorieren von ungewohntem oder unerwünschtem Verhalten (vgl. Tessin, 2011; Bukow, 2001). Stetig muss sich das Individuum entscheiden, ob es sich Situationen oder Kontexten zuwendet oder diese ignoriert. Die Vielfältigkeit an Situationen und Kontexten, mit denen das Individuum in der komplexen Gesellschaft stetig konfrontiert sein kann, bedeutet gleichzeitig aber auch, dass sich die individuellen, latenten und manifesten sozialen Bezüge vervielfältigen. Ein Individuum kann unterschiedlichen sozialen und situativen Zusam- 
menhängen angehören, muss sich von zahlreichen Kontexten aber genauso immer wieder abgrenzen, um sich im komplexen, städtischen Alltag fortbewegen und platzieren zu können und das Leben in einem ambivalenten Stadtteil gestalten zu können.

\section{Vielfaltsverarbeitungsroutinen}

Im Rahmen der Feldforschung hat sich gezeigt, dass die Quartiersbewohner*innen im Alltag in eine Vielzahl unterschiedlicher Relevanzstrukturen, Handlungskontexte und Situationsformate involviert sind, die sie in irgendeiner Form bewältigen müssen. Dieser Bewältigungsprozess gelingt den Quartiersbewohner*innen. Meine Forschung konnte zeigen, dass die Quartiersbewohner*innen - unabhängig von ihrem sozialen Status oder ihrer vermeintlich ,ethnischen“ Zugehörigkeit - auf ihre Weise routiniert situativ mit den Widersprüchen umgehen können, die einem komplexen, diversitätsdurchdrungenen Alltag inhärent sind.

Auf Basis meiner Beobachtungen habe ich in meiner ethnografischen Untersuchung verschiedene Vielfaltsverarbeitungsroutinen herausarbeiten können, von denen insbesondere drei durch eine blasierte Haltung charakterisiert sind. Diese drei Vielfaltsverarbeitungsroutinen sollen im Folgenden detaillierter skizziert werden und in einem weiteren Schritt in ihrer Bedeutung für die postmigrantische Forschung herausgestellt werden.

Grundlegend gehören zu den Vielfaltsverarbeitungsroutinen ganz zentral Abgrenzungspraktiken. Menschen öffnen und verschließen sich unterschiedlichen Situationen und Kontexten gegenüber je nach individuellem Bedarf und Empfinden. Dieser Abgrenzungsprozess geschieht nicht immer bewusst. Die Menschen im Quartier verfolgen individuelle, alltägliche Routinen, mit denen sie Neues und Anderes immer wieder dynamisch einebnen oder an ihren gewohnten Strukturen abprallen lassen und die innerhalb ihres praktischen Bewusstseins quasi unhinterfragt ablaufen, also ins selbstverständliche Verhaltensrepertoire der Individuen eingegangen sind. ${ }^{6}$

Beobachter*in sein: Aus dem imaginierten Schutzraum einer Beobachter*in heraus werden neue soziale Situationen

\footnotetext{
${ }^{6}$ Einen zentralen Wissensbereich des Individuums im Alltag bildet das Routinewissen, auf das das Individuum unhinterfragt und wie selbstverständlich zurückgreift, etwa das Setzen des Blinkers vor dem Abbiegen, die Betätigung des Lichtschalters in der Wohnung, wenn es zu dunkel ist oder aber das Ausweichen vor einer Person, die den eigenen Weg auf dem Bürgersteig kreuzt. Für das Individuum besitzen Routinen eine besondere psychologische Relevanz (vgl. Giddens, 1992). Durch die stetige Reproduktion von Handlungen, also durch Gewohnheit, erlangt das Individuum im Alltag Selbstsicherheit. Alltägliche Gewohnheiten sind der zentrale Referenzpunkt, um „Vertrauen“ in das eigene Handeln und eine ,ontologische Sicherheit“ (Giddens, 1992:116) reproduzieren zu können. Routinen dienen also der Stabilisierung im Alltag.
}

beobachtbar und erfahrbar, ohne dass das Individuum aktiv in die Situation involviert sein muss.

Situationsspezifische Rahmung: Die situationsspezifische Rahmung hat sich als zentraler Mechanismus zur Erklärung und Verarbeitung von Vielfalt und Komplexität herausgestellt. Wahrgenommene soziale Situationen werden als fremdes Setting gerahmt und entsprechend ihrem eigenen (dem anderen) Dunstkreis zugeordnet. Dieser Rahmungsprozess hilft dem Individuum dabei, die Vielzahl an Situationen, die $\mathrm{ihm} / \mathrm{ihr}$ in einem komplexen Alltag begegnen, zu verarbeiten. Im Rückgriff auf den individuellen Wissensvorrat wird dabei für den eigenen Alltag weniger Relevantes mit der notwendigen Distanz betrachtet, relevante Situationen werden hingegen aufgegriffen und aktiv verarbeitet.

Nicht-zu-Ende-Wissen: Es hat sich gezeigt, dass die Menschen den ambivalenten, komplexen und diversitätsdurchdrungenen Alltag als Referenzrahmen nutzen und Situationen und Kontexte nicht vollständig nachvollziehen müssen. Dies impliziert eine gewisse Offenheit und Unabgeschlossenheit gegenüber Neuem und Fremden.

\subsection{Beobachter*in sein:}

IPA: „Unser Wohnzimmerfenster geht direkt aufn Lessingplatz raus von oben, das heißt, wir können super oft einfach von oben gucken: Was passiert da unten? Und wir tun es auch echt oft, wo wir wirklich schon selber denken: Wie alte Leute stehst Du grad am Fenster und guckst da runter. Aber das würd ich aber ja nicht machen, wenn da gar nichts los wär. [Ja] Also, es ist tatsächlich so, wenn Du Bock hast, und es kommt im Fernsehen nichts, da kannst Dich auch mal ans Fenster stellen, da passiert schon genug. Also, das ist wirklich so, also es ist/es spielen sich schon lustige Szenen ab, also, ich finds extrem witzig auf diesen Spielplatz zu gucken [...]“ (I-A, Z. 186-203).

Das Leben in Oberbilk ist durch Widersprüche geprägt, denn Oberbilk ist ein sehr heterogener Stadtteil, in dem unterschiedliche soziale Gruppen (noch) ${ }^{7}$ nebeneinander coexistieren. Mit der Nähe zum Hauptbahnhof, zur Düsseldorfer Innenstadt und zum Naherholungsgebiet Volkgarten, mit zahlreichen Einkaufsmöglichkeiten und den im Stadtteilver-

\footnotetext{
${ }^{7}$ Ziel städtischer Planung ist es häufig öffentliche Räume zu monofunktionalisieren und sie eben nicht mit einem breiten Nutzungsangebot für verschiedene Aktivitäten offen zu halten. Öffentliche Räume verlieren dadurch ihre so wichtige Funktion, nämlich als Alltagsort für alle Nutzergruppen Möglichkeiten der Platzierung zu bieten. Durch die monofunktionelle Nutzung des öffentlichen Raums werden bestimmte Nutzer*innengruppen und ihre Verhaltensweisen systematisch ausgegrenzt. Die (Vor-)Strukturierung von öffentlichen Räumen intendiert bereits eine bestimmte Raumnutzung, die eine subjektive Nutzungsinterpretation verunmöglicht (vgl. dazu Tessin, 2011; Wehrheim, 2009; Klamt, 2006).
} 
gleich noch relativ bezahlbaren Mieten ist Oberbilk für breite Bevölkerungsschichten attraktiv. Die unterschiedlichen Gruppen und Milieus, die im urbanen Raum zusammenkommen, prägen das Stadtbild im öffentlichen Raum. So ist auch die Nutzung des öffentlichen Raumes von der Verschiedenheit seiner Bewohner*innen geprägt. Besonders deutlich wird das, wenn man sich den Lessingplatz als zentralen Ort und Treffpunkt vieler Quartiersbewohner*innen anschaut. Dort kommen Jugendliche, Familien, Trinker*innen, Pädagogi*innen, Sporttreibende, Marktbesucher*innen, Newcomer*innen, Alteingesessene, Arme und Reiche zusammen. In meiner Forschung stellte sich heraus, dass die Menschen, die um den Lessingplatz wohnen, die Vielfalt an Unterschiedlichem und die Irritationen und Störungen, die durch die unterschiedlichen Nutzungsweisen im öffentlichen Raum auftreten, durchaus schätzen und als positiv wahrnehmen. Sie finden es spannend, andere Lebensweise und -stile tagtäglich erleben zu können, auch wenn es immer wieder Situationen gibt, durch die sie sich doch gestört oder irritiert fühlen.

Es zeigte sich, dass sie sich als Beobachter*in durch den öffentlichen Raum bewegen und aus der Beobachter*innenrolle heraus andere, teils fremde Verhaltensweisen aufgreifen oder ignorieren (lernen). Sie nehmen fremde Settings auf dem Platz, etwa die alkoholtrinkenden Stammnutzer*innen oder die ,pöbelnden Jugendlichen“, die manchmal laut sind und stören, zwar wahr, sind durch die Beobachter*innenperspektive aber nicht direkt in die fremde Situation involviert, auch dann nicht, wenn Konflikte ausarten. Die Übernahme der Beobachter*innenrrolle ermöglicht den Stadtbewohner*innen, die Ambivalenzen des Alltags aus einem imaginierten Schutzraum wahrzunehmen. Es ist, wie über den Widersprüchlichkeiten des Lebens zu schweben, ohne unmittelbar davon betroffen zu sein und die Probleme der Anderen lösen zu müssen. Damit verhalten sich die Bewohner*innen neutral blasiert gegenüber Ereignissen im öffentlichen Raum. Fremde Verhaltens- und Handlungsweisen werden von den Quartiersbewohner*innen als Teil eines eigenen Dunstkreises wahr- und hingenommen. Dabei ist es nicht relevant, ob die beobachteten Handlungsweisen im Einzelnen nachvollzogen oder gar verstanden werden können. Die Handlungsweisen erscheinen als folgerichtig innerhalb der Situation, in der sie wahrgenommen werden. Damit haben sie außerhalb der Situation zunächst keine weiterreichende Bedeutung. Konflikte bleiben damit meist situativ begrenzt und müssen demnach in der Regel auch keinen Anlass zur Sorge um den gesamtstädtischen Zusammenhalt geben.

\subsection{Situationsspezifische Rahmung:}

FP 11: „Neben dem Nachbarschaftshaus auf den Bänken sitzen wieder die Stammplatznutzer*innen. Einer von Ihnen hat einen Grill aufgebaut. Der Qualm und Gestank des Grills hängt über dem gesamten Platz. Ich beobachte die Männer eine Zeit lang. Es sind auffällig viele Männer, nur eine Frau sitzt auf der Bank. Die Frau sieht ziemlich fertig und ungepflegt aus. Sie trägt eine weiße dünne Jacke mit Flecken und ich erkenne, dass einer ihrer Schuhe an der Seite aufgerissen ist. Ihre Bewegungen erwecken den Eindruck als sei sie betrunken. Irgendwann, als ich schon nicht mehr richtig hingucke, höre ich laute Stimmen und sehe, wie der Mann, der vorher am Grill stand, die Frau versucht von der Bank wegzuzerren. Die Frau wehrt sich und schreit sehr laut. Der Mann schiebt sie ziemlich grob und aggressiv von der Bank herunter und schleppt sie hinter dem Nachbarschaftshaus davon. Ich denke, dass sie an der anderen Seite des Hauses wieder auftauchen müssten und ich die beiden sehen müsste, aber ich sehe nur, wie der Mann wieder an der Seite der Bänke zum Vorschein kommt und sich zu seinem Grill stellt. Die Frau muss also in die andere Richtung verschwunden sein. Einige Kinder und Jugendliche haben den Lärm wahrgenommen und das Spektakel auch gesehen. Sie schauen zu den Leuten am Nachbarschaftshaus. Nach kurzer Zeit gehen alle zurück an die Tätigkeit, die sie zuvor ausgeübt haben. Die Jugendlichen spielen weiter Fußball. Eine Frau mit Kinderwagen, die scheinbar stehen geblieben ist, als sie den Lärm gehört hat, geht weiter. Einer der Stammplatznutzer*innen geht um die Ecke, um dort hinzupinkeln, wie ich vermute" (FP 11, 23. Mai 2015, Z. 22-38).

Damit verbunden ist die zweite Vielfaltsverarbeitungsroutine der Kontextsetzung und Situationseinordnung, die ich in Anlehnung an die ethnomethodologische Forschung (Garfinkel, 1986; Schütz und Luckmann, 1984; Schütz, 1982; Berger und Luckmann, 2013) herausgearbeitet habe. Menschen ordnen einer Situation bestimmte Ereignisse und Handlungsweisen zu und können die Situation deshalb mental abhaken - auch wenn sie die Handlungsweisen der anderen Personen nicht verstehen. Für das Zusammenleben ist diese Form der intrinsischen Situationseinbettung besonders wichtig, denn durch die Kontextsetzung haben Handlungen einen Rahmen, der situationsadäquates Handeln ermöglicht (vgl. Goffman, 1980). Wie Goffman herausgearbeitet hat, orientieren sich Menschen an Situationen und Kontexten, an Rahmungen. Sie betrachten Momente situationsspezifisch und öffnen oder schließen sich entsprechend der jeweiligen Situation gegenüber. Der blasierte Verhaltensstil kommt in diesem Rahmungsprozess insofern zum Ausdruck, als dass die Quartiersbewohner*innen sich in bestimmten Situationen nicht zuständig fühlen, sie sozusagen situationsspezifisch latente Rollenpositionen einnehmen (Tessin, 2011) und sich zuwenden oder abgrenzen. Irritationen und Widersprüche im Alltag werden entsprechend der jeweiligen Situation, in der sie auftreten, zugeordnet und erscheinen dann als weniger schlimm, bzw. als situationslogisch. Durch die Kausalität und die $\mathrm{Zu}-$ 
ordnung zu einer konkreten Situation sind sie weniger irritierend und damit besser verarbeitbar. Dass dem Individuum bestimmte Konflikte schlichtweg „egal“" sind, ist eine Form der Blasiertheit, ohne die gesellschaftliches Zusammenleben nicht möglich wäre, da es unmöglich ist, sich jeder irritierenden Situation hinzuwenden. Natürlich bedeutet das Abgrenzen können und dürfen aber auch, dass sich das Individuum aus Situationen herausnehmen kann, in denen ein Eingreifen bzw. Handeln eigentlich hilfreich gewesen wäre. Eine ins Extreme gesteigerte Blasiertheit kann dann auch negative Folgen für das Zusammenleben der Stadtbewohner*innen haben: Das Individuum grenzt sich ab und zeigt sich teilnahmslos gegenüber seinem Umfeld. Im schlimmsten Fall stumpft es so sehr ab, dass es Missstände und Konflikte, die im alltäglichen Leben sichtbar werden, unbeteiligt an sich abprallen lässt. Mangelnde Empathie und zunehmendes Desinteresse an den Lebensumständen anderer können die Folge sein. Man denke hier an den Vorfall in einer Essener Bankfiliale 2017, als mehrere Bankkund*innen über einen offensichtlich bewusstlosen Mann stiegen, ohne ihm zu helfen (vgl. u.a. SZ, 2017). Der blasierte Verhaltensstil und die Selbstverständlichkeit Wegschauen zu können schützt also auch davor, sich für Situationen verantwortlich zu fühlen, in denen ein Eingreifen und Handeln angebracht gewesen wäre. In diesem Spannungsfeld zwischen Situationsinvolviertheit und Distanzeinnehmen bewegt sich das Individuum im urbanen Alltag schlichtweg und es liegt bei ihm, das richtige $\mathrm{Maß}$ an Teilnahme zu finden.

\subsection{Nicht-zu-Ende-Wissen:}

IPA: „Ich find's halt gut, wenn du einfach aus der Tür gehst oder von a nach $b$ und auf dem Weg von a nach $b$ passieren irgendwie drei Dinge wo du denkst Wat ist jetzt hier gerade passiert so was war das denn und dann denkst du irgendwie drüber nach oder eben nicht, aber wenn du drüber nachdenkst so ja ne dann war das wohl innerhalb dieses Dunstkreises wo das halt grad passiert ist, war es wohl völlig folgerichtig und du verstehst es gerade gar nicht aber es wird schon seinen Sinn haben“ (I-A, Z. 294-299).

Die Situationsrahmung, bzw. das Abhaken einer Situation als Teil eines fremden Dunstkreises, erlaubt dem Individuum darüber hinaus ein Nicht-zu-Ende-Wissen. Dies bedeutet, dass dem Kern der beobachteten/erlebten Situation nicht auf den Grund gegangen werden muss, bzw. dass es nicht weiter schlimm ist, wenn die jeweiligen Handlungspraktiken der anderen Situationsteilnehmenden nicht gänzlich nachvollzogen werden können. Im Rahmen der Forschung zeigte sich, dass der konkret erlebte, diversitätsdurchdrungene Alltag als Referenzrahmen für die Bewertung von Situationen hinzugezogen wird und als Bewertungsgrundlage eine Offenheit gegenüber Situationen zulässt.
Das alltägliche Leben in einem durch Diversität geprägten Stadtteil ermöglicht eine gewisse Gleichgültigkeit gegenüber Diversität, also gegenüber fremden Situationen und Ereignissen. Dies hängt zunächst mit der Komplexität des Alltags zusammen, der aus Unvorhersehbarkeiten besteht. Es ist trivial normal, dass in einem diversitätsgeprägten Quartier Dinge und Ereignisse auftreten, die das Individuum nicht gänzlich nachvollziehen kann. Eben dieses Wissen um Vielfalt trägt, so zeigte sich, mit dazu bei, dass die Bewohner*innen in ihren Kategoriebildungsprozessen nicht festgefahren sind. Auch wenn sozialpsychologisch immer davon ausgegangen wird, dass das Individuum sich Dinge erklärbar machen muss, bzw. dass es Menschen, Verhaltensweisen und Artefakte bestimmten Kategorien zuweist, um sie zu verstehen, so zeigte sich, dass die alltäglich erlebte Vielfalt eine Durchlässigkeit zwischen den verschiedenen Kategorien erzeugt und damit einem vorurteilsbeladenen Schubladendenken entgegenwirkt. Natürlich bleiben Kategorien und Denkmuster im individuellen Wissensvorrat als Leitlinien für das alltägliche Leben bestehen, sie sind aber nicht fest und statisch, sondern flüssig und können sich im Laufe der Zeit verformen und verändern.

Die Kategorie „urbaner komplexer Alltag“ reicht aus, um Dingen eine nicht-Definierbarkeit zu erlauben und sie im Dauerablauf des Alltags ${ }^{8}$ als normales und nicht hinterfragtes Phänomen zu akzeptieren. Dies deutet darauf hin, dass es eine besondere Errungenschaft der Städter*innen ist, überhaupt blasiert sein zu können und zu dürfen, denn diese Form der Gleichgültigkeit ist die Basis für Offenheit und Toleranz. Die Quartiersbewohner*innen handeln entsprechend diversitätssensibel und inklusiv, wenn sie blasiert handeln, da sie auf diese Weise andere Lebensstile und -weisen existieren lassen.

\section{Was bedeuten diese Überlegungen für das Zusammenleben in der postmigrantischen Stadt?}

Die Menschen im Quartier sind gleich im anders sein. Sie bedienen sich an der Kraft des Alltags und reproduzieren den Alltag dauerhaft dynamisch, um ihr Leben ihren Bedürfnissen entsprechend organisieren zu können. An dieser Stelle soll zunächst festgehalten werden, dass die Praktiken der tagtäglichen Ambivalenzbewältigung auf eine äußerst diversitätssensible Grundpragmatik der Stadtbewohner*innen verweisen, die sich inklusionsorientiert durch den urbanen Raum bewegen. Die Vielfaltsverarbeitungsroutinen und die

\footnotetext{
${ }^{8}$ In Anlehnung an Bukow (2010) ist mit dem Begriff Dauerablauf des Alltags gemeint, dass der Alltag des Einzelnen, aber auch der Quartiersalltag insgesamt durch die unterschiedlichen, sich teils stetig reproduzierenden Handlungsweisen der Menschen ständig am Leben erhalten werden. Es ist sozusagen eine Bedürfnisgrundlage des Menschen, den Alltag in einem routinierten Dauerablauf zu erhalten, was nicht bedeutet, dass der Alltag nicht wandelbar ist, vielmehr kann Neues und Fremdes latent und Schritt für Schritt eingeebnet werden (vgl. dazu u.a. Giddens, 1992; Goffman, 1980)
} 
Ausbildung eines blasierten Verhaltensstils machen deutlich, dass die Quartiersbewohner*innen versuchen, Störendes und Irritierendes in den Dauerablauf des Alltags einzuebnen, um sich adäquat im komplexen Alltag fortbewegen zu können. Damit handeln sie aus einer Alltagpragmatik heraus, die das Funktionieren und eben nicht das Nicht-Funktionieren des diversitätsgeprägten Zusammenlebens als Handlungsmaxime hat. Desintegrativen Ansätzen und Sichtweisen auf das urbane Zusammenleben in der Stadt kann auf Basis dieser Ergebnisse ein grundlegend diversitätssensibler Umgang mit Stadtgesellschaft entgegen gestellt werden. Die alltagpraktische Verarbeitung von Vielfalt und Komplexität erfolgt mittels individueller Abgrenzungspraktiken, die gleichzeitig Inklusionspraktiken darstellen. Abgrenzungspraktiken sind damit ebenso ein konstitutiver Teil städtischer Realität. Die Fähigkeit zur Abgrenzung bedeutet gleichzeitig Inklusion, da die Stadtbewohner*innen durch ihre Abgrenzung das $\mathrm{Zu}-$ sammenleben und den Dauerablauf des Alltags immer wieder am Funktionieren halten. Die Anerkennung der Abgrenzungspraktiken als inklusives Verhaltensrepertoire der Städter*innen kann sowohl für die wissenschaftliche als auch die politische Diskussion vielversprechend sein. Denn im Gegenteil zur defizitären Perspektive, verdeutlicht der Blick auf die inklusiven Abgrenzungspraktiken, in welchem Maße Menschen sich ohne Eingriffe von außen miteinander arrangieren können. Die Abgrenzung des Individuums als inklusive Handlungspraktik zu betrachten, hilft, das Zusammenleben zu verstehen und adäquat zu bewerten.

Für eine postmigrantische Forschung bedeutet dies, dass die Menschen nicht entlang ihrer vermeintlich „ethnischen“, religiösen oder generationalen (sogenannte zweite oder dritte Zuwandergeneration) zugewiesenen Zugehörigkeiten betrachtet werden, sondern entlang ihrer Praktiken, die sie im urbanen Raum zur Platzierung und Verortung anwenden. Vor dem Hintergrund dieser an den pragmatischen Alltagsaktivitäten orientierten Praktiken spielt das ,,migrantisch sein" keine Rolle, sondern man ist schlichtweg Quartiersbewohner*in. Damit wird nicht der Mensch mit seinen zugeschriebenen Eigenschaften zur Betrachtungsgrundlage, sondern die Praktiken in einem wie auch immer gearteten, individuell umgrenzten Raum, dem Quartier. Mit dem Quartier als Referenzrahmen und der Beobachtung von im Alltag vollzogenen Praktiken umgeht man also stigmatisierenden, diskriminierenden und essentialisierenden Zuschreibungen, da man die Menschen von einer „gleichen“ Basis aus betrachtet. Zusammengenommen sind die genannten Vielfaltsverarbeitungsroutinen Grenzziehungen, die in einem stetigen, individuell unterschiedlichen Balanceakt zwischen $N \ddot{a}$ he und Distanz und Öffnen und Schließen vollzogen werden. Es sind Grenzziehungen, die das Individuum ausführen muss, um sich adäquat im Alltag bewegen zu können und um sich seiner Selbst vergewissern zu können.

Die Simmelsche Blasiertheit und die damit verbundene Nassehische Distanz und Gleichgültigkeit als inklusive Praxis anzuerkennen, bedeutet auch, tradierte Vorstellun- gen von gesellschaftlichem Zusammenleben zu überdenken. Nähe und allumfassende Vergemeinschaftung dürfen nicht die ausschließlichen städteplanerischen oder pädagogischen Handlungsziele sein. Vergemeinschaftung findet meist situativ statt und wird im nächsten Moment durch andere, neue Situationen und Konstellationen wieder aufgebrochen. Vor diesem Hintergrund ist es wichtig, tradierte lokale (stadtund sozialpolitische-) Maßnahmen und Programme im Hinblick darauf zu überdenken, inwieweit sie dazu geeignet sind, alle Quartiersbewohner*innen einzubinden. Nicht eine alles umwölbende, gemeinschaftsbasierte Imagination sollte Maßnahmen und Programme leiten, sondern höchstens Formen situativer Vergemeinschaftung, die im nächsten Moment wieder aufgebrochen werden können und die Raum für das „Unterschiedlich-Sein“ und „Unterschiedlich-Sein-Dürfen“ lassen. Distanz und Grenzziehungen und damit eine gewisse blasierte Haltung sind wichtige Ressourcen, um nebeneinander co-existieren zu können.

Datenverfüg barkeit. Die empirische Grundlage dieses Beitrages bilden teilnarrative und leitfadengestuitzte Interviews sowie Gesprächsprotokolle mit Menschen im Quartier, die sensible, zum Teil personenbezogene Daten enthalten. Da den Interviewpartner*innen Anonymität und Vertraulichkeit zugesichert wurden, sind die Transkripte nicht öffentlich zugänglich. Informationen zum Datenmaterial können bei der Autorin erfragt werden.

Interessenkonflikt. Die Autorin erklärt, dass kein Interessenkonflikt besteht.

Danksagung. Mein besonderer Dank gilt den Interviewpartner*innen und allen Menschen, die meine Arbeit über die letzten Jahre hinweg unterstützt haben. Den Gutachter*innen sowie Karin Wiest danke ich außerdem für ihre konstruktiven Überarbeitungshinweise.

Begutachtung. This paper was edited by Karin Wiest and reviewed by two anonymous referees.

\section{Literatur}

Bahrdt, H. P.: Entstädterung in der Urbanisierung. Soziologische Gedanken zum Städtebau von morgen, Baukunst und Werkform, Bd. 12, 653-657, 1956.

Baumgärtner, E.: Lokalität und kulturelle Heterogenität, transcript, Berlin, 2009.

Berding, N.: Perspektiven auf das Zusammenleben im Quartier Das Beispiel Düsseldorf Oberbilk, in: Die kompakte Stadt der Zukunft. Auf dem Weg zu einer inklusiven und nachhaltigen Stadtgesellschaft, Herausgeber: Berding, N., Bukow, W.-D. und Cudak, K., Springer VS, Wiesbaden, 105-126, 2018 a. 
Berding, N.: Der urbane Raum Lessingplatz in DüsseldorfOberbilk. Städtischen Alltag arrangieren: Eine ethnografische Studie über ganz alltägliche Konflikte im Umgang mit urbaner Vielfalt. Unveröffentliche Dissertation, Universität zu Köln, 2018 b.

Berger, P. L. and Luckmann, T.: Die gesellschaftliche Konstruktion der Wirklichkeit. Eine Theorie der Wissenssoziologie, Fischer Taschenbuch Verlag, Frankfurt a. M., 2013.

Bukow, W.-D.: Urbanes Zusammenleben. Zum Umgang mit Migration und Mobilität in europäischen Stadtgesellschaften, Springer VS Wiesbaden, 2010.

Bukow, W.-D. and Yildiz, E.: Der Umgang mit der Stadtgesellschaft. Ist die multikulturelle Stadt gescheitert oder wird sie zu einem Erfolgsmodell?, Leske + Budrich, Opladen, 2002.

Bukow, W.-D., Nikodem, C., Schulze, E., and Yildiz, E. (Hrsg.): Die multikulturelle Stadt. Von der Selbstverständlichkeit im städtischen Alltag, Leske + Budrich, Opladen, 2001.

Bürger- und Schützenzeitung: Düsseldorf Oberbilk ab 1927-1939 (ab 1935 nur noch Bürgerzeitung)

Dangschat, J.: Soziale Mischung - (k)ein Ruhekissen!?, in: Migration \& Integration 3 - Dialog zwischen Politik, Wissenschaft und Praxis, Herausgeber: Biffl, G., Bad Vöslau, Omninum KG, 175$185,2013$.

Dika, A., Jeitler, B., Krasny, E., and Širbegović, A.: Balkanmeile, 24 Stunden Ottakringer Straße. Lokale Identitäten und globale Transformationsprozesse; ein Reiseführer aus Wien, Turia + Kant, Wien, 2011.

Express: Rund um Ellerstraße Marokkaner verzweifeln: „Diese Leute machen uns kaputt“, online aufrufbar: https://www.express.de/duesseldorf/rund-um-ellerstrassemarokkaner-verzweifeln-diese-leute-machen-uns-kaputt23412228 (letzter Zugriff: 10.01.2019), 14. Juni 2016.

FAZ: Marokkanische Straftäter: „Wer die Absicht hat, die Willkommenskultur $\mathrm{zu}$ missbrauchen...", online aufrufbar: http://www.faz.net/aktuell/politik/fluechtlingskrise/marokkanischestraftaeter-wer-die-absicht-hat-die-willkommenskultur-zumissbrauchen-14035190.html (letzter Zugriff: 26.06.2018), 26. Februar 2016.

Feldtkeller, A.: Zur Alltagstauglichkeit unserer Städte. Wechselwirkungen zwischen Städtebau und täglichem Handeln, Schiller, Berlin, 2012.

Foroutan, N.: Postmigrantische Gesellschaften, in: Einwanderungsgesellschaft Deutschland, Herausgeber: Brinkmann, H. and Sauer, M., Springer VS, Wiesbaden, 2016.

Garfinkel, H.: Ethnomethodological studies of work, Routledge \& Kegan Paul, London, 1986.

Glebe, G.: Oberbilk - ein innerstädtisches Industrie- und Arbeiterviertel im Industriezeitalter, in: Lokale Transformationsprozesse in der Global City. Düsseldorf - Oberbilk - Strukturwandel eines Stadtteils, Herausgeber: Glebe, G. and Schneider, H., Düsseldorf, Düsseldorfer Geographische Schriften, 59-86, 1998.

Giddens, A.: Die Konstitution der Gesellschaft, Campus Verlag, Frankfurt a. M., 1992.

Goffman, E.: Verhalten in sozialen Situationen. Strukturen und Regeln der Interaktion im öffentlichen Raum, Verlagsgruppe Bertelsmann, Gütersloh, 1971.

Goffman, E.: Rahmen-Analyse - Ein Versuch über die Organisation von Alltagserfahrungen, Suhrkamp, Frankfurt a. M., 1980.
Harlander, T., Kuhn, G., and Wüstenrot Stiftung: Soziale Mischung in der Stadt. Case Studies - Wohnungspolitik in Europa - Historische Analyse, Kraemer Verlag, Stuttgart/Zürich, 2012.

Häußermann, H.: Desintegration durch Stadtpolitik?, online aufrufbar: http://www.bpb.de/apuz/29492/ desintegration-durch-stadtpolitik (letzter Zugriff: 10.06.2017), 2006.

Heckmann, F.: Ethnische Kolonien: Schonraum für Integration oder Verstärker der Ausgrenzung?, in: Ghettos oder ethnische Kolonien? Entwicklungschancen von Stadtteilen mit hohem Zuwandereranteil, Herausgeber: Forschungsinstitut der Friedrich-EbertStiftung, Abt. Arbeit und Sozialpolitik, Bonn, 29-42, 1998.

Heide, A., Krasny, E., and Babina, L.: Aufbruch in die Nähe, Wien Lerchenfelder Strasse, Turia + Kant, Wien, 2010.

Heitmeyer, W., Dollase, R., and Backes, O. (Hrsg.): Die Krise der Städte - Analysen zu den Folgen desintegrativer Stadtentwicklung für das ethnisch-kulturelle Zusammenleben, Suhrkamp, Frankfurt a. M., 1998.

IBA-Studie: Das Leitbild von der „Urbanen Mischung“, Geschichte, Stand der Forschung, Ein- und Ausblick, Berlin, online aufrufbar: http://www.stadtentwicklung.berlin.de/staedtH\T1

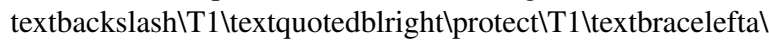

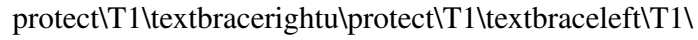
textbackslashss \protect\T1\textbracerightebau/baukultur/iba/ download/studien/IBA-Studie_Urbane_Mischung.pdf (letzter Zugriff: 10.06.2018), 2013.

Klamt, M.: Raum und Norm. Zum Verhalten und seiner Regulierung in verschiedenen öffentlichen Räumen, in: Öffentliche Räume - öffentliche Träume. Zur Kontroverse über die Stadt und die Gesellschaft, Herausgeber: Wiegandt, C., Lit Verlag, Berlin, 2945, 2006.

Lanz, S.: Berlin aufgemischt: abendländisch, multikulturell, kosmopolitisch? Die politische Konstruktion einer Einwanderungsstadt, transcript, Frankfurt a. d. O., Bielefeld, 2007.

Lanz, S.: Die ganze Welt der Stadt im Blick: Versuche einer pluralisierten kritischen Stadtforschung. Unveröffentlichte Habilitationsschrift, Europa-Universität Viadrina Frankfurt a. d. O., 2016.

Lindner, W.: Jugendliche in der Stadt: Im Spannungsfeld von Devianz (-Phantasien) und urbaner Kompetenz, in: Der Umgang mit der Stadtgesellschaft. Ist die multikulturelle Stadt gescheitert oder wird sie zu einem Erfolgsmodell?, Herausgeber: Bukow, W.-D. and Yildiz, E., Leske + Budrich, Opladen, 217-239, 2002.

Mecheril, P., Dirim, İ., Kalpaka, A., Melter, and do Mar Castro Varela, M. (Hrsg.): Migrationspädagogik, Beltz, Basel, Weinheim, 2010.

Nassehi, A.: Das stahlharte Gehäuse der Zugehörigkeit. Unschärfen im Diskurs um die multikulturelle Gesellschaft, in: Nation, Ethnie, Minderheit. Beiträge zur Aktualität ethnischer Konflikte, Herausgeber: Nassehi, A., Böhlau, Köln, Weimar, Wien, 177208, 1997.

Nassehi, A.: Gut, dass wir uns fremd geworden sind, online aufrufbar: https://www.welt.de/debatte/kommentare/article6491106/ Gut-dass-wir-uns-fremd-geworden-sind.html (letzter Zugriff: 15.06.2018), 2017a.

Nassehi, A.: Kein Editorial. Urbanität als Anerkennungsmedium, in: Kursbuch 190, Herausgeber: Nassehi, A. and Felixberger, P., Stadt, Ansichten, Muhrmann Verlag, Hamburg, 3-9, 2017 b. 
Oltmer, J.: Migrationsregime vor Ort und lokales Aushandeln von Migration: Springer VS, Wiesbaden, 2017.

Ottersbach, M. and Yildiz, E.: Migration in der metropolitanen Gesellschaft. Zwischen Ethnisierung und globaler Neuorientierung, LIT Verlag, Münster, 2004.

Radtke, F.-O.: Lob der Gleichgültigkeit. Die Konstruktion der Fremden im Diskurs des Multikulturalismus, in: Das Eigene und das Fremde. Neuer Rassismus in der Alten Welt, Herausgeber: Bielefeld, U., Junius, Hamburg, 79-97, 1991,

Römhild, R.: Global Heimat. Der Alltag junger Migranten in den Widersprüchen der Einwanderungsgesellschaft, in: Neue Vielfalt in der urbanen Stadtgesellschaft, Herausgeber: Bukow, W.-D., Heck, G., Schulze, E., and Yildiz, E., Springer VS, Wiesbaden, 21-32, 2011.

Römhild, R.: Diversität?! Postethnische Perspektiven für eine reflexive Migrationsforschung, in: Kultur, Gesellschaft, Migration: Die reflexive Wende in der Migrationsforschung, Herausgeber: Nieswand, B. and Drotbohm, H., Springer VS, Wiesbaden, 255$270,2014$.

Römhild, R. and Bergmann, S.: global heimat. Ethnographische Recherchen im transnationalen Frankfurt. Kulturanthropologie Notizen, Frankfurt a. M., 2003.

Ronneberger, K. and Tsianos, V.: Panische Räume. Das Ghetto und die „Parallelgesellschaft“, in: No integration?! Kulturwissenschaftliche Beiträge zur Integrationsdebatte in Europa, Herausgeber: Hess, S., Binder, J., and Moser, J., transcript, Bielefeld, 137-152, 2009.

RP (Rheinische Post): Polizei stört kriminelle Wohlfühlzone, online aufrufbar: https://rp-online.de/nrw/staedte/duesseldorf/razziaim-maghreb-viertel-in-duesseldorf-polizei-stoert-die-

wohlfuehlzone_aid-9214387 (letzter Zugriff: 07.08.2018), 17. Januar 2016.

Schneider, R.: Die Utopie der sozialen Mischung. Gleichheit ist beim Wohnen in der Stadt nicht durchzusetzen, online aufrufbar: http://www.deutschlandradiokultur.de/ gesellschaft-die-utopie-der-sozialen-mischung.1005.de.html? dram:article_id=273800 (letzter Zugriff: 15.06.2018), 2016.

Schöller-Schwedes, O.: Der Stadtsoziologe Georg Simmel - Ein Missverständnis und seine Folgen, Berliner Journal für Soziologie, 18, 649-662, 2008.

Schütz, A.: Das Problem der Relevanz, Suhrkamp, Frankfurt a.M., 1982.

Schütz, A. and Luckmann, T.: Strukturen der Lebenswelt Band 1 Suhrkamp, Frankfurt a.M., 1979, Band 2 - Suhrkamp, Frankfurt a.M., 1984.

Sennett, R.: Verfall und Ende des öffentlichen Lebens. Die Tyrannei der Intimität, Suhrkamp, Frankfurt a.M., 2013.
Simmel, G.: Die Großstädte und das Geistesleben, Suhrkamp, Frankfurt a.M., 2006.

SZ (Süddeutsche Zeitung): Sterbenden Rentner in Bank ignoriert: Prozess startet, online aufrufbar: https://www.sueddeutsche.de/news/panorama/prozessesterbenden-rentner-in-bank-ignoriert-prozess-startet-dpa.urnnewsml-dpa-com-20090101-170917-99-83946 (letzter Zugriff: 10.02.2018), 17. September 2017.

Terkessidis, M.: Andere Räume, Räume der Anderen, Bildpunkt. Zeitschrift der IG Bildende Kunst: Raumgreifen, 24-27, online aufrufbar: https://www.linksnet.de/artikel/20435 (letzter Zugriff: 10.06.2018), 2005.

Tessin, W.: Freiraum und Verhalten. Soziologische Aspekte der Nutzung und Planung städtischer Freiräume, Springer VS, Wiesbaden, 2011.

Tsianos, V.: Urbane Paniken. Zur Entstehung eines antimuslimischen Urbanismus, in: Wer macht Demo_kratie? Kritische Beiträge zu Migration und Machtverhältnissen, Herausgeber: Duygu, G., Zülfukar, Ç., and Allmende, E. V., edition assemblage, Münster, 22-42, 2013.

Vaiou, D. and Kalandides, A.: Cities of "others": public space and everyday practices, Geogr. Helv., 64, 11-20, https://doi.org/10.5194/gh-64-11-2009, 2009.

Wehrheim, J.: Der Fremde und die Ordnung der Räume, Budrich + Leske, Opladen, 2009.

Wiesemann, L.: Öffentliche Räume und Diversität. Geographien der Begegnung in einem migrationsgeprägten Quartier - das Beispiel Köln-Mülheim, Lit Verlag, Berlin, 2015.

Wirth, L.: Urbanität als Lebensform - Soziale Prozesse in der Stadt, in: Stadt- und Sozialstruktur. Arbeiten zur sozialen Segregation, Ghettobildung und Stadtplanung: dreizehn Aufsätze, Herausgeber: Herlyn, U., Nymphenburger Verlagshandlung, München, 42-66, 1974.

Yildiz, E.: Die weltoffene Stadt. Wie Migration Globalisierung zum urbanen Alltag macht, transcript, Bielefeld, 2014.

Yildiz, E.: Das Quartier als Experimentierwelt einer inklusiven Stadt, in: Die kompakte Stadt der Zukunft. Auf dem Weg zu einer inklusiven und nachhaltigen Stadtgesellschaft, Herausgeber: Berding, N., Bukow, W.-D., and Cudak, K., Springer VS, Wiesbaden, 159-180, 2018.

Yildiz, E. and Hill, M.: Nach der Migration. Postmigrantische Perspektiven jenseits der Parallelgesellschaft, Bielefeld, transcript Verlag, 2015. 